\title{
Study about upper limb on highly repetitive work in maquila operations
}

\author{
Lopez-Millan Francisco ; De la Vega, Enrique ${ }^{\mathrm{b}}$; Rodriguez, Manuel ${ }^{\mathrm{a}}$; Ayala, Armando ${ }^{\mathrm{a}}$ \\ ${ }^{a}$ Instituto Tecnologico de Cd. Juarez, División de Estudios de Posgrado e Investigación, Ave. \\ Tecnológico No. 1340 C.P. 32500 Ciudad Juárez, Chih. México \\ ${ }^{b}$ Instituo Tecnologico de Hermosillo, División de Estudios de Posgrado e Investigación, Ave. \\ Tecnológico s/n. CP 83170, Hermosillo, Sonora, México
}

\begin{abstract}
Industrial work is a very active sector in the economy of countries; an important part of people's work is done using the upper extremities. The purpose of this project is to characterize the effect of upper extremity work, analyze its relationship with the hand strength and the presence of fatigue and develop a model with the ability to estimate recovery times for the shoulder using variables different from the biomechanical variables.
\end{abstract}

Keywords: fatigue, recovery time, biomechanical modeling, shoulder pain

\section{Introduction.}

Labor in maquila operations is an important economic contributor, especially for those countries considering emerging economies, México for instance. Maquila is a manufacturing plant which production is made basically to export, US mostly of times. There are a lot of operations doing by hands and using all kind of manual tools; that is why is necessary conduct a research about repetitive work to know the implications on fatigue and recovery time and to characterize movements of upper limb, focused on shoulder area.

Human kind has evolved for a millions of years until today, in the time line appear industrial revolution which changed drastically how product are made and put into consumer hands, consequently labor has increases upon time resulting on more people exposed to repetitive work effects, thus human body systems was not either design or evolved for this work condition, [1] that is why musculoskeletal disorders (MSD) finally appear.

Occupational studies about risk of musculoskeletal disorders as a result of frequent work exposure has been conducted since long ago, that it's "were recognized as having occupational etiologic factors as early as the beginning of the 18th century, however, it was not until the 1970s that occupational factors were examined using epidemiologic methods, and the work-relatedness of these conditions began appearing regularly in the international scientific literature" [2]. MSD such as low back pain and wrist trauma has been in the spotlight due to costs and occurrence but disorders and pain complains has been raised for shoulder area, [3]. Shoulder disorders are not easy to classify probably due to the multifactorial nature of causes. MSD [4] are related with degenerative and inflammatory conditions affecting muscles, tendons, joints, ligaments, commonly involved this body regions; wrist, low back, neck, shoulder.

However data about incidence and prevalence of MSD are not easy to obtain even when MSD are widespread in many countries with all negative consequences, [4].

Conditions like those are frequently present in maquila activities. The purpose of the study is to find relationships between occupational activities involving upper limb, personal characteristics, force behavior and perceived fatigue. 


\section{Method.}

As a main purpose on this research was considered real work conditions, so all data has been collected from assembly line; it is not an easy process to get into plant facilities. In order to obtain the most valuable information, a very intensive operations involving hand and arms was choose, confidentiality is needed to kept on company name and logos. It is highly appreciated all support they gave to this research.

First part of the study was data collection, consisted on measured some anthropometrics for both hands; Length, width, height, width of the wrist, height of the wrist, height of the thumb, width of the thumb, height of the middle finger and width of the middle finger. The other anthropometric data were height and weight. Additionally handgrip and finger pinch grip force was measure with a Jamar(C) hand dynamometer and finger dynamometer. Every day, 3 measurements are made every half hour, from the sixth hour until the eight hour, for handgrip and finger grip for each hand. A perceived fatigue mapping for hand and shoulder was applied at the end of the journey. They questioned about remained presence of pain, numbness or stiffness once people return back home. Scale was simply; 1 for occasionally, 2 for often and 3 correspond to always.

Every job was analyzed using 3DSSPPC software and recovery time obtained with Rohmert formula. Multiple linear regression was used to analyze relationship between force and anthropometrics. The repetitive measurements general model was used to analyze the force within hours and within days. Once data were obtained and analyzed, the last part of the research consisted in try to modeling fatigue due to repetitive work.

\section{Results}

One purpose of the study was to find relationships between personal and occupational characteristics related with fatigue. Once data were obtained, first a principal component analysis was conducted to find groups of variables, exerted force of the hand is response variable. After several iterations, two groups was formed; one for hand anthropometrics and other for personal anthropometrics. Since there are relationships between variables, using a lineal regression analysis obtained an $R$ coefficient equals to .804 , showing a strong relationship between anthropometrics and hand force. Once found this relationship, the next step was to relation hand force with time, assuming that hand force decreases in a function of time, [5] presented a method of analysis of fatigue on the basis of a reduction in the force exerted may be a good index of fatigue induced by repetitive work. A repetitive measurements general model was used to test force behavior within day of the week and within hour of the journey. There was found not enough statistical evidence to conclude that hand force decrease while the work journey or weekdays advance. However, perceived fatigue results show high notes for hand and shoulder. Here is important to describe what was the work studied, it was 26 work stations, all people were women; in maquila high percent of labor are woman. All they are sitting, cycle times are about 4 seconds per job, mostly operations are made whit very intensive hand work, that is; pinch grips, inserting connectors, twisting wires, pushing with thumbs and packaging. Shoulder remains static while hand is doing the job. All work stations are not adjustable on height, which means all furniture is fixed, seats and tables. It is notorious that many of employees are wearing some kind of tape around fingers.

Answers about remained symptoms like pain, numbness or stiffness once they are back in home are the next: $61 \%$ mentioned that occasionally recognize al least one of symptoms and a $36 \%$ mentioned that always recognize al least one of symptoms. That is, $97 \%$ of woman labor is affected by the very intensive job.

The next was relation additional variables in a different way. Each job was biomechanical modeled using 3DSSPPC software, shoulder muscle groups shows to be the most affected, then a Rohmert recovery time analysis [6] was made for each job. 3DSSPPC showed static workload and calculate the moment for different body muscle groups, based on anthropometrics calculate an average and a standard deviation for each muscle group moment. Rohmert analysis requires force moment and duration of the exposure or exertion. Exertion time becomes significant over 15 seconds.

After several combinations, a lineal regression model was found, considering recovery time as response variable, predictors were; height, weight, posture angle for shoulder, exertion time for shoulder and perceived fatigue. Model obtained result on an $\mathbf{R}$ value of .879 which shows the strength relationship among variables. The model is the follow:

RecoveryTime $=-67.19+17.53 *$ Height $0.16 *$ Weight $+0.592 *$ ShoulderAngle + $.598 *$ Perceived Fatigue $+1.647 *$ Exertion time 
WinBUGSC software was used to optimized the model using Bayesian statistical analysis and Monte Carlo Markov Chains simulation. For Bayesian analysis the obtained linear regression model is assumed as prior information. A goodness of fit test shows a very good approach to a Normal distribution for all the variables of model. So, all the model parameters were considered as prior normal. After 10,000 iterations for each parameter, the resulting model is the follow:

Recovery Time $=-59.41+12.53 *$ Height -
$0.1522 *$ Weight $+0.588 *$ Shoulder Angle
$0.611 *$ Perceived Fatigue $+1.638 *$ Exertion time

A comparative between recovery time obtained using Rohmert formula and recovery time obtained using the optimized model shows a difference of 0.463 , it is close to zero, the ideal difference.

\section{Discussion.}

Very intensive work seems to have no effect in force behavior as a function of time, however at the end of the day, people showed signs of fatigue trough pain and numbness. It conducts to conclude that repetitive work really affect upper limb, for the study, shoulder group of muscles. An additional relevant finding is that upper limb works in two dimensions, a physiological one for the wrist, when very highly repetitive work is done, movements are in a pace for about 350 times per hour. But, due to the workstation design and to the repetitive work, shoulder remains in a same posture, which means in a static posture while wrist and hand do the job. It is a static workload in shoulder area, both sides.

Recovery time is a kind of shadow price, remain hidden. In industrial time studies, additional time is provided to compensate fatigue. Recovery time has another meaning, it is the time provided to a group of muscles to let recuperation for static load effect. It may work as follow; in work design every time a muscle is affected by the static load the recovery come returning the muscle group to a neutral position, but the job could be continuing, it is no necessary to stop working, it is not affection to efficiency and productivity. Including recovery time could be a great strategy to lower the risk on MSD, for the case, a high repetitive production system in combination with sustained work postures could lead to shoulder pain.

How the model works? Recovery time estimation needs for a tool like 3DSSPPC and the understanding of Rohmert formula. The proposal model for recovery time needs the data included in regression model, that is, height given en centimeters, weight, given en kilograms, the shoulder angle in sustained posture, an interview with operators quantified between 1 and 3 and finally the time on sustained posture. All data are introduced in formula and the output is recovery time. It use is simple too, for the amount of time in sustained posture is necessary to give the amount of time for recuperation of muscle group.

It is important to remark that force moment for the wrist are not easy to calculate, specially considering that moment is in function of distance, an distance in hands trend to be shorts, additionally sustained posture, according to the research findings, is not common in wrist, it is common to the fingers. In that sense, a different strategy should be used for intensive work in hands, may be used stretch exercises for instance.

Finally, it is remarkable in the observation of repetitive work, how are present two basic dimensions of job, one for the wrist-fingers which work dynamically and one for the shoulder working statically. Make sense having different strategies for the same upper limb.

\section{References}

[1] Bernard, B. Thomas, (1997). "Musculoskeletal Disorders and Workplace Factors; A Critical Review of Epidemiologic Evidence for Work-Related Musculoskeletal Disorders of the Neck, Upper Extremity, and Low Back". Centers for Disease Control and Prevention, National Institute for Occupational Safety and Health (NIOSH).

[2] Kumar, Shrawan (2001) 'Theories of musculoskeletal injury causation', Ergonomics, 44:1, $17-47$

[3] Sommerich, C.M. McGlothin, J.D. Marras, W.S. (1993). Occupational risk factors associated with soft tissue disorders: a review of recent investigations in the literature. Ergonomics, Vol. 36, No. 6, 697-717.

[4] Punnett, L. Wegman, D. H. (2004). Work-related musculoskeletal disorders: the epidemiologic evidence and the debate. Journal of Electromyography and Kinesiology 14, 13-23.

[5] Rohmert Walter (1973). "Problems in determining rest allowances". Applied Ergonomics, 4.2 91-95.

[6] Roman-Liu, Danuta, Tokarski, Tomasz and Kowalewski, Radosaw(2005). Decrease of force capabilities as an index of upper limb fatigue', Ergonomics,48:8,930 948 